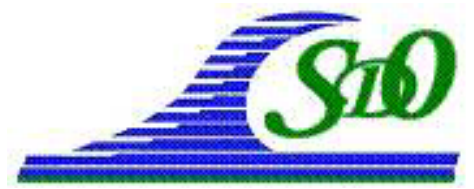

XI ìmes Journées Nationales Génie Côtier - Génie Civil

Les Sables d'Olonne, 22-25 juin 2010

DOI:10.5150/jngcgc.2010.041-L C Editions Paralia CFL

disponible en ligne - http://www.paralia.fr - available online

\title{
Sédimentation et ostréiculture dans l'estuaire du Payré (Vendée, France) : apports de la caractérisation physico- chimique des sédiments
}

\section{Cécile LE GUERN ${ }^{1}$, Pierre CONIL ${ }^{1}$, Cyril MANCEAU ${ }^{1}$, Daniel LEVACHER ${ }^{2}$}

1. BRGM, Service Géologique Régional des Pays de la Loire,

1 rue des Saumonières, BP 92342, 44323 Nantes Cedex, France.

c.leguern@brgm.fr

2. Université de Caen, Faculté des Sciences, UMR 6143 CNRS - M2C,

Esplanade de la Paix, 14032 Caen, France.

daniel.levacher@unicaen.fr

\section{Résumé :}

L'activité ostréicole s'est développée dans l'estuaire du Payré au niveau de la confluence de ses différents affluents. Afin d'évaluer l'état de l'érosion - sédimentation sur la partie basse du bassin versant en lien avec les problèmes d'envasement et d'ensablement rencontrés par les ostréiculteurs, une campagne d'analyse des sédiments superficiels a été réalisée. Vingt-neuf prélèvements sont localisés en zone influencée par la marée dynamique et 4 points restants sont en dehors de son influence.

Les analyses granulométriques réalisées ont permis d'obtenir des informations précieuses sur le fonctionnement hydrologique et sédimentaire de l'estuaire. En effet, les différents faciès ont pu être individualisés et les zones d'influences marines et continentales délimitées. Les teneurs en matière organique ainsi que les analyses chimiques des éléments majeurs et des éléments traces (ICP multi-élémentaire), ont par la suite permis de statuer sur le caractère potentiellement polluant de certains de ces sédiments. La présence d'arsenic, en chrome et en baryum en quantité significative a pu être expliquée par deux hypothèses : le fond géochimique local qui semble l'hypothèse la plus probable et la présence d'activités industrielles pouvant être à leur origine. Des teneurs importantes en phosphates ont également été identifiées.

Mots-clés :

Génie côtier - Sédiments - Environnement littoral - Estuaire - Payré - Vendée France - Ecosystèmes côtiers - Erosion - Sédimentation

\section{Introduction}

L'activité ostréicole s'est développée dans l'estuaire du Payré au niveau de la confluence de ses différents affluents. Face aux problématiques d'envasement et d'ensablement des parcs à huîtres auxquelles font face les ostréiculteurs, il s'avère nécessaire de comprendre la dynamique hydrologique qui contrôle la mise en place des différents faciès de sédimentation rencontrés sur les bords et au fond des berges. A cette 
fin, l'origine et la nature des matériaux rencontrés dans cet estuaire doivent être préalablement mises en évidence, de même que l'existence et la localisation des zones d'érosion et de sédimentation.

La caractérisation physico-chimique des sédiments superficiels a ainsi été employée comme outil pour aider à évaluer l'état de l'érosion / sédimentation sur la partie basse du bassin versant en lien avec les problèmes rencontrés par les ostréiculteurs.

\section{Description du bassin versant du Payré}

\subsection{Contexte géographique}

Situé en Vendée à l'extrémité sud du Massif armoricain, le Payré est un petit cours d'eau encadré par les villes des Sables d'Olonne au Nord et de La Tranche sur Mer au Sud. Il s'écoule sur une distance d'environ vingt kilomètres selon une direction Nord Est-Sud Ouest et se jette dans l'océan Atlantique à proximité de la ville de Talmont Saint Hilaire.

Son cours peut être décomposé en trois affluents principaux alimentés par quelques ruisseaux. Le chenal du Payré est le plus important et coule depuis le massif granitique d'Avrillé situé au Nord-Ouest et jusqu'à Talmont (figure 1). Sur l'un de ses affluents, le Châtenay, se situe le barrage de Finfarine. Cette retenue d'eau est utilisée pour l'alimentation en eau potable. Le second affluent, plus petit, appelé "le petit chenal des hautes mers", se situe à l'ouest du précédent. Le dernier bras, au sud, est le ruisseau de l'île Bernard. Ces trois affluents se rejoignent dans les marais en zone topographiquement basse, puis se jettent dans l'océan au niveau d'un havre. La superficie du bassin versant est de $154 \mathrm{~km}^{2}$.

L'occupation des sols est marquée par de nombreuses activités anthropiques, dont les intérêts peuvent être divergents en matière de gestion de l'eau. L'agriculture (terres cultivées, prairies, vignes...) et la conchyliculture sont ainsi prépondérantes. Le bassin présente en outre des activités industrielles et de services et des activités touristiques. L'urbanisation est, comme dans la plupart des zones côtières, en développement.

\subsection{Contexte géologique}

Le bassin versant du Payré se situe sur des terrains très variés, témoignant de l'histoire géologique complexe du Talmondais. Celle-ci s'inscrit dans le contexte plus vaste de l'histoire du Massif armoricain et plus précisément de son segment vendéen.

Deux principaux ensembles géologiques se distinguent sur le bassin versant (figure 1) :

- Au nord et à l'est de Talmont, affleurent principalement des terrains de socle d'origine plutonique et métamorphique (extrémité Sud du Massif Armoricain). Constitués de schistes et micaschistes orientés N130 recoupés par le granite d'Avrillé, ils correspondent aux zones topographiquement hautes. 


\section{XI $I^{\text {èmes }}$ Journées Nationales Génie Côtier - Génie Civil \\ Les Sables d'Olonne, 22-25 juin 2010}

- Au sud de Talmont, affleurent des terrains sédimentaires principalement d'âge Jurassique (bordure septentrionale du bassin d'Aquitaine) discordants sur les terrains de socle précédents. Constitués notamment de calcaires, ils correspondent aux zones topographiquement plus basses.

Enfin, de nombreuses séries d'âge Quaternaire recouvrent les vallées du bassin versant d'une mince pellicule.

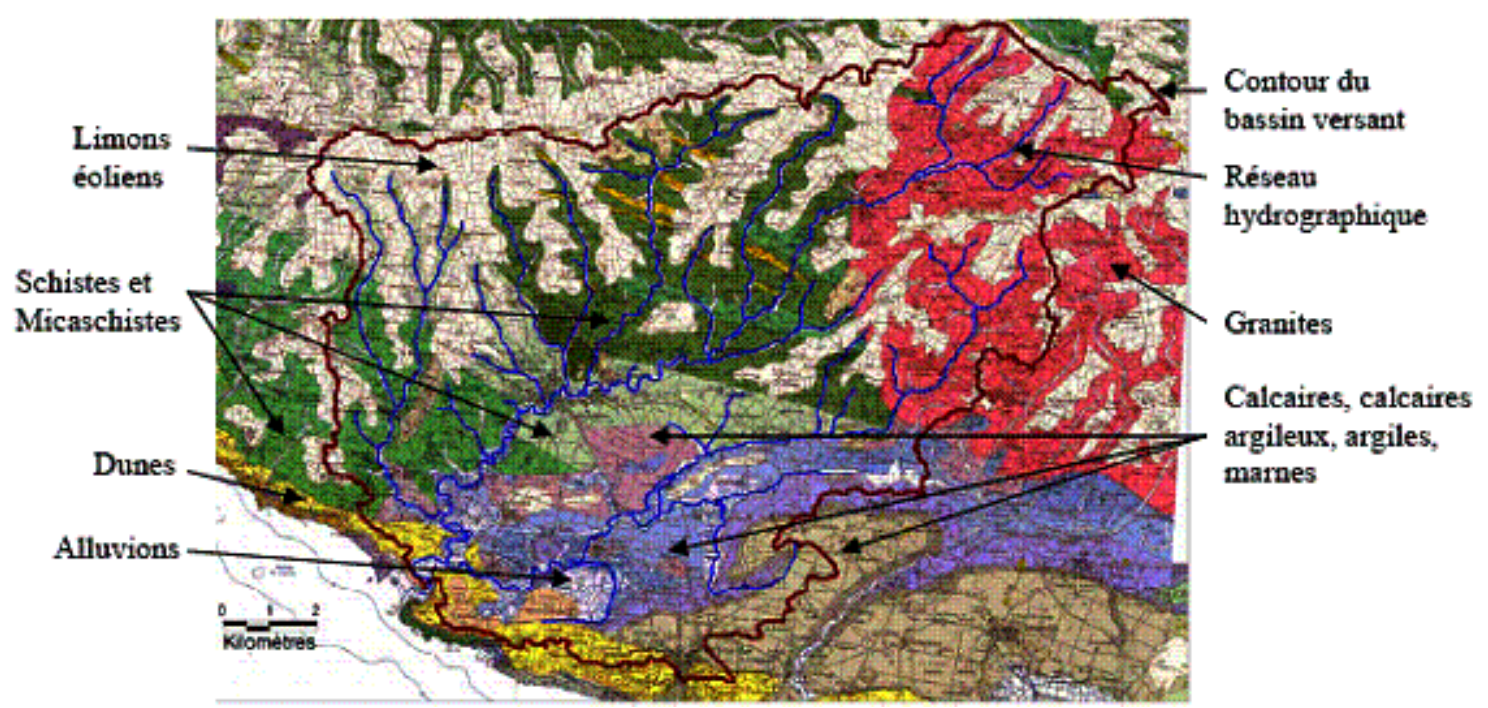

Figure 1. Carte géologique de la région de Talmont Saint Hilaire, Extrait de la carte au 1/50000 eme des Sables d'Olonne-Longeville (TERS \& GABILLY, 1986).

\section{Matériels et méthodes}

\subsection{Prélèvements}

La figure 2 présente les 33 points de prélèvements sédimentaires superficiels. 29 de ces points sont localisés en zone influencée par la marée dynamique et les 4 points restants sont en dehors de son influence. Les prélèvements ont été effectués à la tarière à main, sur une profondeur de $20 \mathrm{~cm}$.

\subsection{Mesures et analyses}

Les analyses granulométriques ainsi que les teneurs en matière organique ont été déterminées sur l'ensemble des échantillons prélevés. Les teneurs en carbonates et les compositions chimiques en éléments majeurs et traces ont été analysées sur 7 échantillons $(1,2,13,15,16,18,23)$. Parmi les 7 points sélectionnés, 5 représentent des points d'entrée dans le système estuarien et 2 sont proches de sources potentielles de pollution :

- Les points $\mathrm{n}^{\circ} 1$ et 2 , localisés le plus à l'aval de l'estuaire, sont destinés à évaluer s'il y a ou non entrée d'éléments d'origine marine. 
- Les points $n^{\circ} 13,16$ et 23 visent à évaluer les apports à l'estuaire de chacun des trois sous bassin versant du Payré.

- Le point $n^{\circ} 15$ est localisé à l'aval des villages de la Dagoterie et de la Doubletière (pas de tout à l'égout).

- Le point $\mathrm{n}^{\circ} 18$ est situé à une vingtaine de mètres à l'aval du point de rejet des eaux traitées par la station d'épuration de la SAUR.

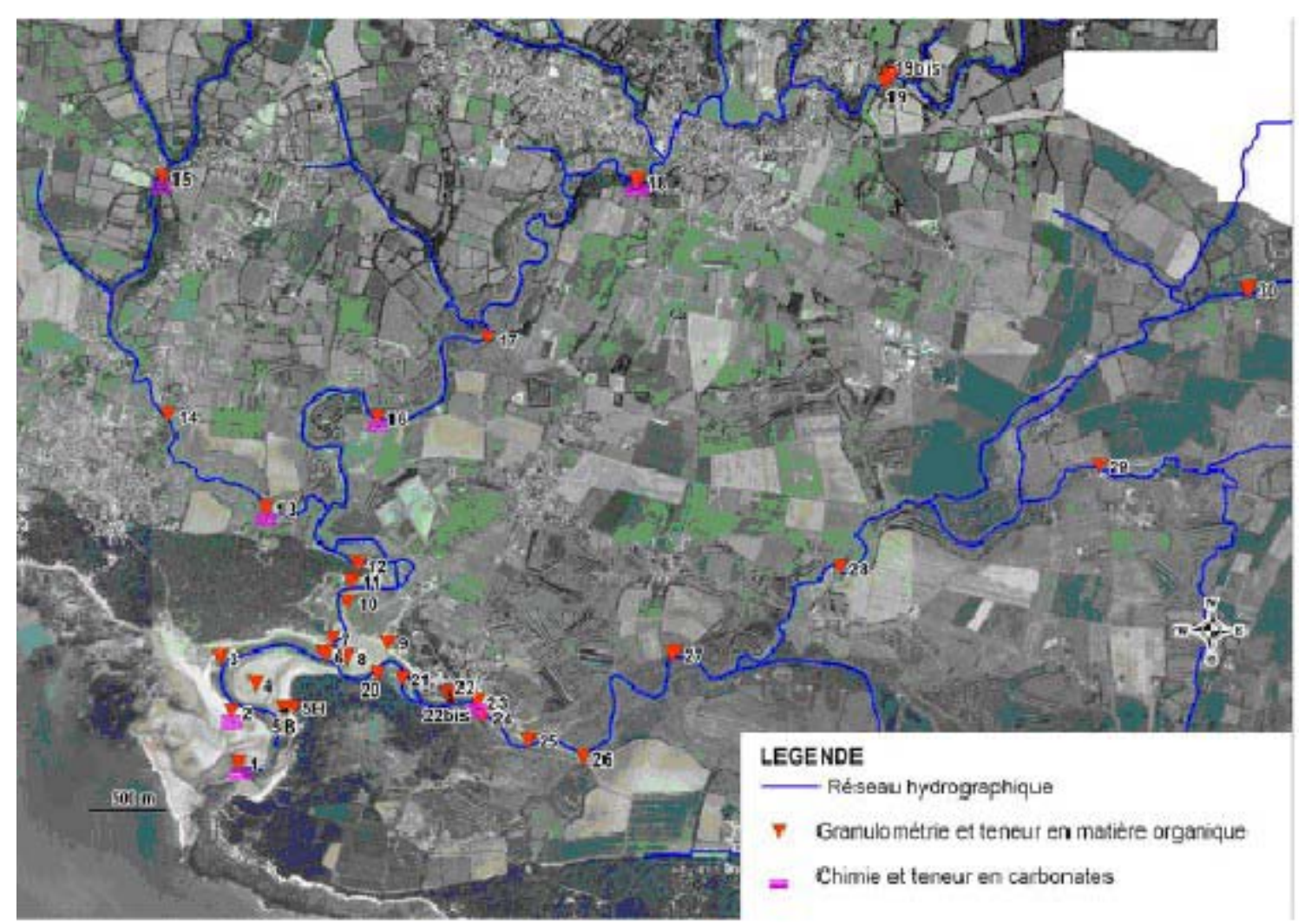

Figure 2. Localisation des points de prélèvements de sédiments superficiels.

\section{- Granulométrie}

Les analyses granulométriques ont été réalisées par la méthode optique de granulométrie laser (granulomètre BECKMAN COULTER). Chaque échantillon a fait l'objet d'un prélèvement aliquote dilué ensuite dans environ $10 \mathrm{ml}$ d'eau, puis homogénéisé sous agitation magnétique. La suspension obtenue a ensuite été tamisée en voie humide à $2 \mathrm{~mm}$. Le refus de tamis (supérieur à $2 \mathrm{~mm}$ ), non pris en compte par le granulomètre laser, a été séparé et comptabilisé à part et seule la fraction inférieure à 2 $\mathrm{mm}$ a été soumise à l'analyse optique. Le sédiment restant a ensuite été dilué afin d'obtenir les pourcentages d'obscuration permettant d'acquérir des résultats corrects.

- Matières organiques

Le protocole consiste à éliminer la matière organique avec du peroxyde d'hydrogène concentré (eau oxygénée, concentration 33\% volume) et à déduire la masse 
correspondante par différence entre la masse d'échantillon avant et après traitement. Le traitement à l'eau oxygénée est effectué sur 1 à $3 \mathrm{~g}$ d'échantillon séché à $35^{\circ} \mathrm{C}$.

- Eléments traces métalliques

Les échantillons ont été analysés selon les normes en vigueur (NF ISO 11464, NFX 31-147 et XP 33-012). Les protocoles MO077 et MO111 ont été appliqués. Les échantillons ont donc été séchés à $40^{\circ} \mathrm{C}$. Ensuite, seule la fraction inférieure à $2 \mathrm{~mm}$ a été prise en compte. 34 éléments ont été dosés par ICP multi-élémentaire dont 8 éléments majeurs et 26 éléments traces.

\section{Résultats et discussion}

\subsection{Analyse sédimentologique}

Les analyses granulométriques (figure 3) ont permis de mettre en évidence deux types de faciès sédimentaires : des sables à l'aval et des vases argileuses à l'amont. Une observation complémentaire à la loupe binoculaire a été réalisée pour déterminer l'origine des particules.

L'ensemble sableux est caractérisé par un mode principal d'environ $300 \mu \mathrm{m}$, un mode secondaire d'environ $700 \mu \mathrm{m}$ (figure 4) composé de débris coquilliers. Une origine marine des particules est ainsi montrée. La taille moyenne des particules décroit de l'aval vers l'amont, le tri granulométrique s'améliore de l'aval vers l'amont. L'ensemble sableux remonte plus haut le long du bras Sud du Payré (entre les points 23 et 24). Les points 3 et 7 se démarquent de l'ensemble par une sédimentation fine due à leur localisation dans une zone de calme hydrodynamique favorisant la décantation des particules fines.

L'ensemble vaseux est caractérisé par un mode principal compris entre 5 et $30 \mu \mathrm{m}$. L'origine continentale des particules apparaît logique dans la mesure où les apports fluviaux amènent des particules fines à l'estuaire. La sédimentation apparaît homogène le long des trois affluents majeurs. Le bras de Talmont montre néanmoins une sédimentation plus fine vers l'aval.

Concernant les parcs ostréicoles, le Port La Guittière constitue une zone de mélange entre les particules fines d'origine continentale et les particules sableuses d'origine marine. La sédimentation près des concessions implantées à proximité du Veillon apparaît essentiellement d'origine continentale.

\subsection{Origine des teneurs en matière organique}

L'ensemble des sédiments situés dans l'estuaire montre des teneurs très faibles $(>0,7 \%)$. Cette observation peut être expliquée d'une part par la nature sableuse des particules peu favorables au développement ou au piégeage de matière organique et d'autre part par un contexte hydrodynamique de dépôt peu favorable à la sédimentation de particules 
Thème 2 -Dynamique sédimentaire et transports des particules

organiques (légères). Seul l'échantillon $\mathrm{n}^{\circ} 7$ possède une concentration en matière organique de près de $4 \%$, expliquée par la nature abritée et vaseuse de la zone.

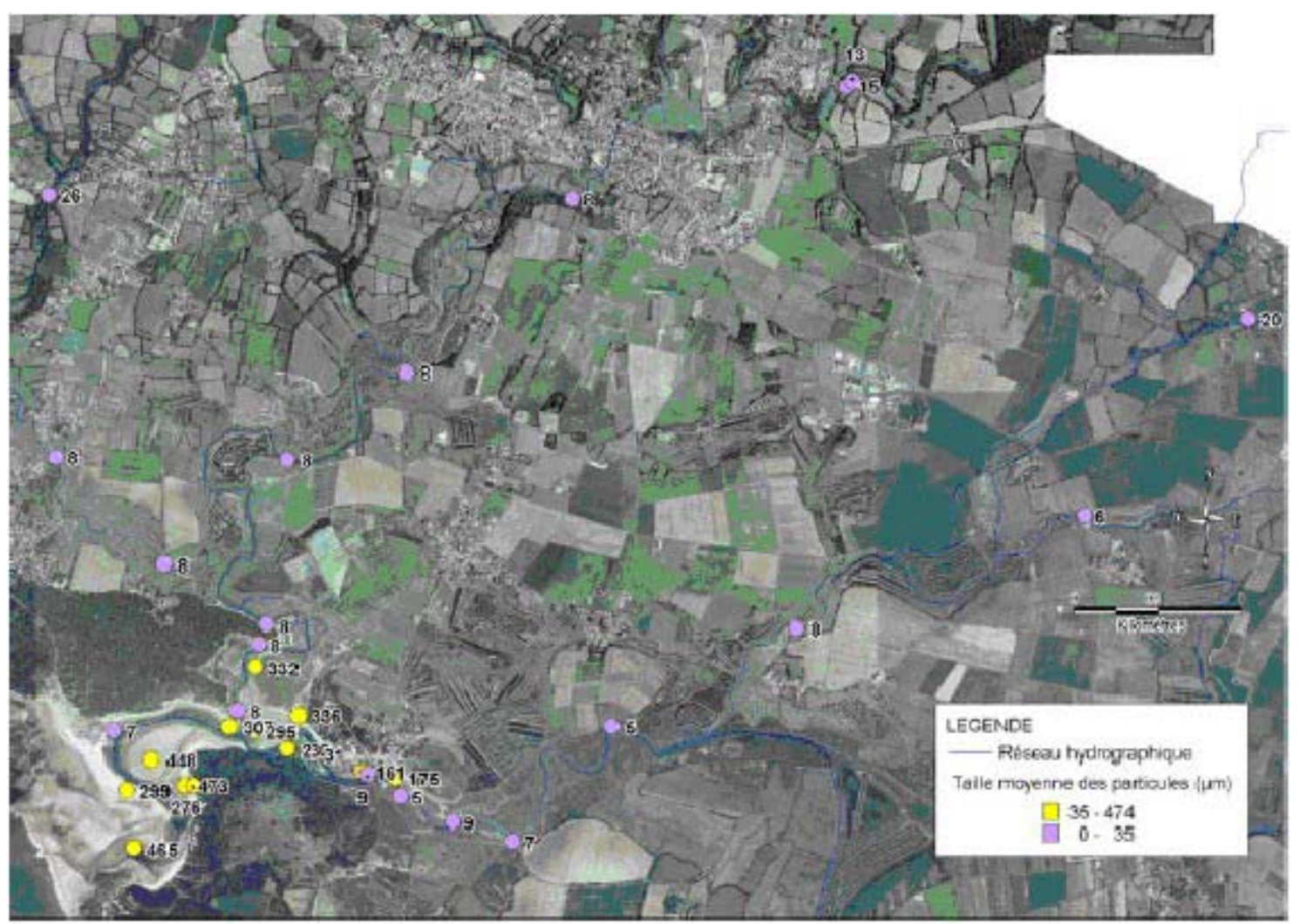

Figure 3. Répartition des différents faciès sédimentaires rencontrés sur la zone d'étude (sables marins en jaune, vases argileuses continentales en violet) et taille moyenne des particules.

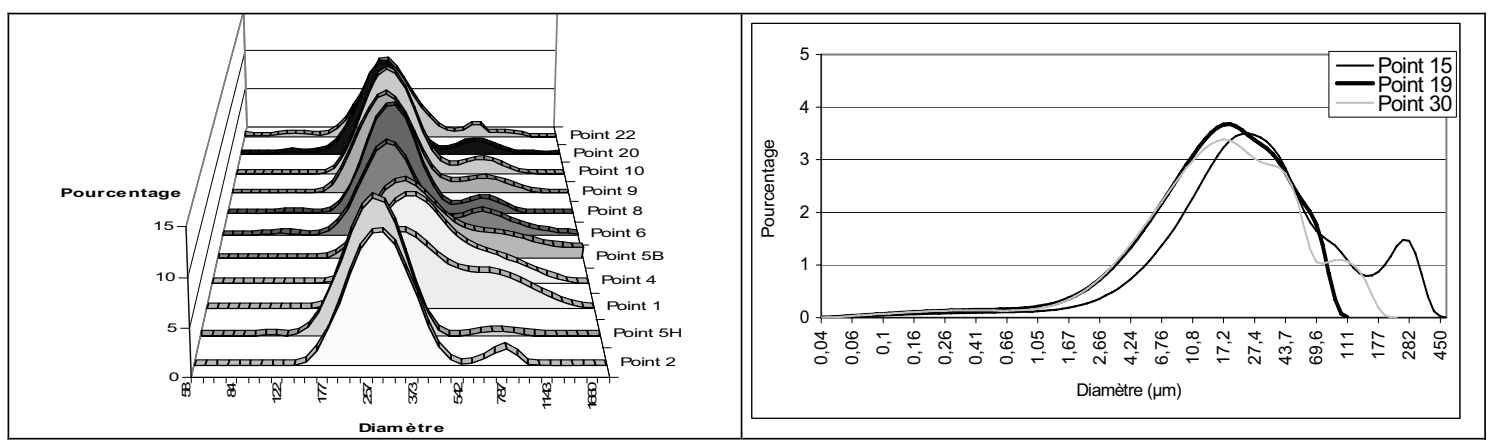

Figure 4. Courbes granulométriques des points de l'ensemble sableux (à gauche) et des points de l'ensemble vaseux (à droite).

Les sédiments récoltés le long des 3 affluents montrent des teneurs en matière organique plus élevées (généralement comprises entre 1,5 et $5 \%$ ). Une partie de ces teneurs 


\section{XI $I^{\text {emes }}$ Journées Nationales Génie Côtier - Génie Civil \\ Les Sables d'Olonne, 22-25 juin 2010}

pourrait témoigner d'une influence anthropique par les eaux usées (fosses septiques, station d'épuration).

Enfin, les points non pris en compte précédemment et qui sont situés en dehors de la zone d'influence marine montrent les concentrations les plus fortes $(19,98 \%, 8,29 \%$ et $6,62 \%$ ). La teneur de près de $20 \%$ observée au point n ${ }^{\circ} 15$ montre bien l'influence locale des rejets. Les deux autres teneurs peuvent quant à elles témoigner d'une production locale plus importante du fait de la nature davantage terrigène des particules.

\subsection{Origine des éléments trace métalliques et du phosphate}

Parmi les différents éléments analysés, le baryum, le chrome et l'arsenic ressortent avec des teneurs moyennes élevées (391, 69 et $59 \mathrm{mg} / \mathrm{kg}$ respectivement), (figure 5).

Ces teneurs élevées peuvent être mises en relation avec le fond géochimique régional. La carte géologique $\mathrm{n}^{\circ} 584$ des Sables d'Olonne montre la présence de minerai de plomb argentifère au Lieu-dit La Mine à proximité de l'estuaire du Payré. L'étude des minéralisations par le BRGM dans les années 80 a montré l'existence d'un horizon de 2 à 3 mètres de puissance. Il est composé de barytine et de sulfures (pyrite) pouvant expliquer la présence de baryum, de chrome et d'arsenic dans les sédiments estuariens. Peu d'activités anthropiques semblent en revanche pouvoir être à l'origine des teneurs observées.

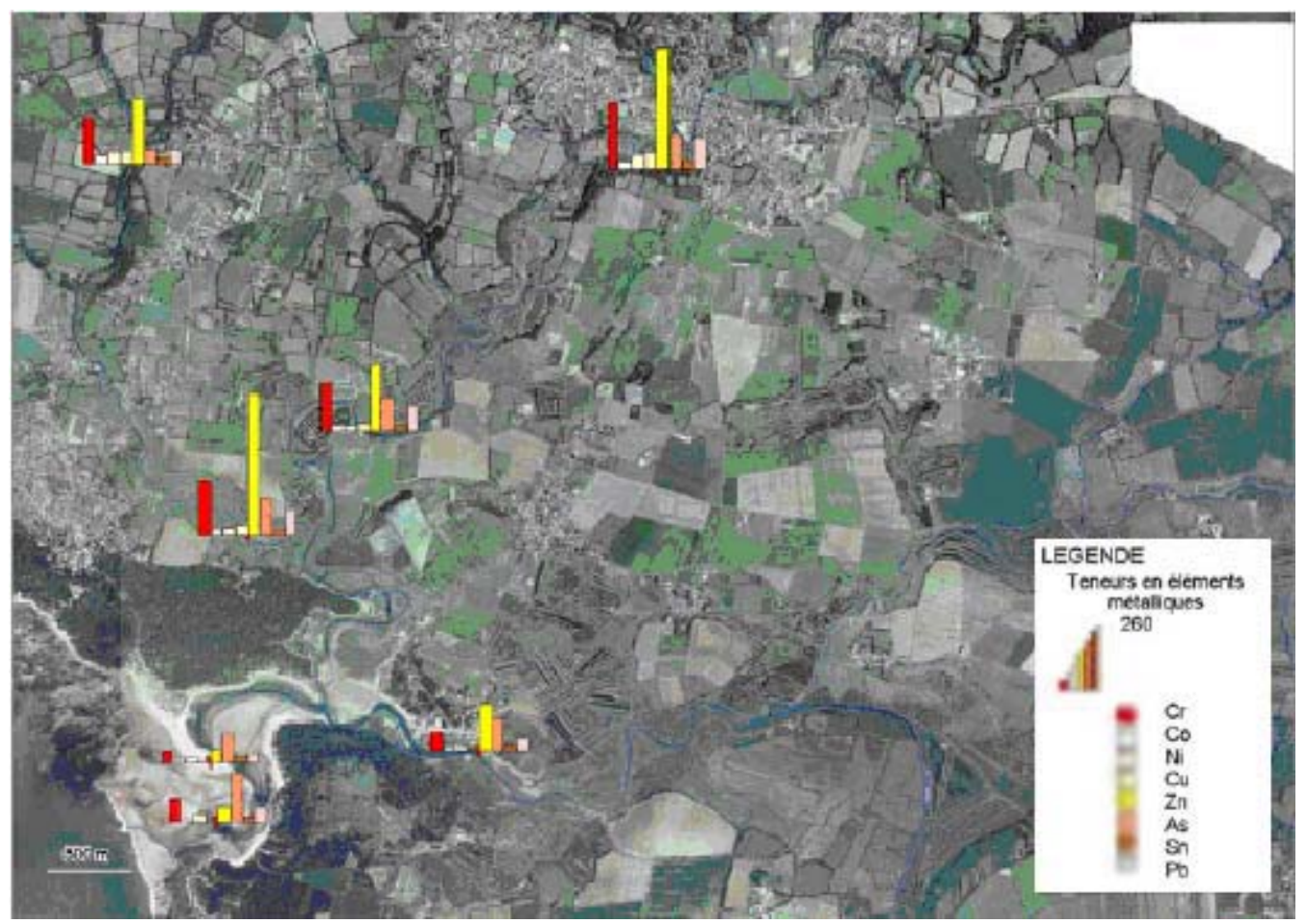

Figure 5. Teneurs en éléments trace métalliques $(\mathrm{mg} / \mathrm{kg})$ dans les 7 sédiments de berge analysés. 
Les deux sous-bassins versant des Hautes mers et du Payré (Bras Ouest et bras de Talmont) montrent par ailleurs des concentrations en phosphates (>1600 ppm) très supérieures à celles observées sur le bras Sud $(963 \mathrm{ppm})$ et sur les points d'entrée marine (640 et $653 \mathrm{ppm}$ ). Un pic à plus de $3600 \mathrm{ppm}$ est même observé en aval du rejet de la station d'épuration. Ainsi, les concentrations les plus importantes sont observées à l'amont de l'estuaire. Elles diminuent ensuite vers l'aval.

Deux hypothèses peuvent expliquer ce phénomène ; soit les phosphates sont dilués vers l'estuaire extérieur, soit ils sont consommés par des organismes tels que les algues. La consommation d'oxygène engendrée par cette seconde hypothèse pourrait perturber l'activité ostréicole.

\section{Conclusions}

La caractérisation des sédiments superficiels apparaît pertinente pour évaluer l'état de l'érosion / sédimentation sur la partie basse du bassin versant en lien avec les problèmes rencontrés par les ostréiculteurs. Les analyses granulométriques réalisées ont ainsi permis d'obtenir des informations précieuses sur le fonctionnement hydrologique et sédimentaire de l'estuaire. En effet, les différents faciès ont pu être individualisés de même que les zones d'influences marines et continentales ont pu être délimitées.

Les analyses chimiques ont permis d'émettre des hypothèses sur l'origine de certains polluants. Ainsi, les teneurs en matière organique apparaissent par endroit particulièrement influencées par les rejets anthropiques. Les teneurs significatives en arsenic, en chrome et en baryum observées peuvent être expliquées en revanche principalement par le fond géochimique local. Des teneurs importantes en phosphates ont également été identifiées.

\section{Références bibliographiques}

TERS M., GABILLY J. (1986). Carte géol. France (1/50000), feuille Les Sablesd'Olonne/Longeville (584). Orléans : BRGM - Notice explicative par Goujou J.C., Debrand-Passard S., Hantzpergue P., Lebret P. (1994), 95 p. 